\title{
Clinical Nuclear Medicine
}

H.-J. Biersack and L.M. Freeman, eds.

New York, NY: Springer, 2007, 548 pages, \$279

The modern era of nuclear medicine imaging and therapy is well into its seventh decade, and many textbooks have been published during this era. One of the more recent books has the same title as the book under review. Edited by Cook, Maisey, Britton, and Chengazi and published by Hodder Arnold in 2007, that Clinical Nuclear Medicine has a distinctly European flavor.

This Clinical Nuclear Medicine is a unique European and American joint venture that focuses on the universal state of the art in both diagnostic and therapeutic radionuclide methodology. The 30 chapters are almost equally split between 16 American and 48 European authors. In addition, 2 chapters were contributed by authors from Taiwan and Canada. This book is organized into 3 parts: basic sciences, nuclear medicine diagnosis, and nuclear medicine therapy. The part on basic science has 2 chapters concisely dealing with physics, instrumentation, radiation protection, radiochemistry, and radiopharmacy. The part on nuclear medicine diagnosis has 18 chapters covering the different organ systems, lymphoma, infection, PET in oncology, pediatric imaging, and miscellaneous scans. The part on nuclear medicine therapy has 10 chapters covering thyroid diseases, bone metastases, receptor therapy, neuroendocrine tumors, radioimmunotherapy, hepatic tumors, pleural and peritoneal effusions, radiosynoviorthesis, and miscellaneous disorders.
This book emphasizes pertinent clinical applications rather than attempting to cover everything that would be included in a large, comprehensive textbook. Therefore, the information helps with optimization of patient care by enabling the reader to select the proper radiopharmaceuticals and the proper techniques or approaches to clinical procedures. A unique feature of this textbook is that it incorporates PET into each organ system or disease-specific chapter rather than presenting PET as a separate chapter.

This book is easily readable, with presentation of essential points and plentiful references, and the quality of the figures and tables is excellent. The aim was not to create a classic textbook but a compilation of currently relevant topics in clinical nuclear medicine. This practical book is a useful guide to students, technologists, and practitioners in nuclear medicine and radiology, as well as to general physicians who request nuclear diagnosis or treatment. Clinical Nuclear Medicine certainly will be a welcome contribution to the bookshelf of nuclear medicine or radiology clinics.

COPYRIGHT ( 2008 by the Society of Nuclear Medicine, Inc. DOI: 10.2967/jnumed.108.050393 